CONFORMAL GEOMETRY AND DYNAMICS

An Electronic Journal of the American Mathematical Society

Volume 7, Pages 1-10 (January 23, 2003)

S $1088-4173(03) 00088-2$

\title{
DISTORTION IN THE SPHERICAL METRIC UNDER QUASICONFORMAL MAPPINGS
}

\author{
PETER A. HÄSTÖ
}

\begin{abstract}
This paper contains bounds for the distortion in the spherical metric, that is to say, bounds for the constant of Hölder continuity of mappings $f:\left(\mathbb{R}^{n}, q\right) \rightarrow\left(\mathbb{R}^{n}, q\right)$ where $q$ denotes the spherical metric. The mappings considered are $K$-quasiconformal $(K \geq 1)$ and satisfy some normalizations or restrictions. All bounds are explicit and asymptotically sharp as $K \rightarrow 1$.
\end{abstract}

\section{INTRODUCTION}

In this paper we derive explicit asymptotically sharp estimates for the constant of Hölder continuity in the spherical metric of quasiconformal (qc) mappings. These results are based on reducing the problem to the Euclidean metric case, where Hölder continuity is well-known. The paper 3] provides explicit estimates with some nice properties (cf. Lemma 2.5) that will be shown to hold for the constants in the spherical metric also.

A similar, but more restricted, result due to Petra Bonfert-Taylor has previously appeared as Lemma 4.1 of [2] (cited as Lemma 2.6] in this paper). This result gives an estimate for the Hölder constant with respect to the spherical metric of planar quasiconformal mappings, which, however, is not asymptotically sharp as $K \rightarrow 1$.

This paper is organized as follows: in the next section the main results are stated, in Section 3 some standard notation is reviewed and explicit estimates pertaining to qc mappings are presented. Section 4 contains various lemmas and the proof of the main results. In Section 5 an explicit bound for the Hölder constant in the spherical metric of $K$-quasisymmetric real functions is derived.

\section{MAIN RESULTS}

We first introduce some notation and terminology for the formulation of our main results. For a more complete account, see the following section.

Definition 2.1. We define $M_{i}$ (for $R \geq 1$ ) to be the smallest constant such that the following inequalities hold for all $x, y \in B^{n}(R)$ and for all $K$-quasiconformal mappings $f: \mathbb{R}^{n} \rightarrow \mathbb{R}^{n}$, with $n \geq 2, f(0)=0$ that satisfy the additional conditions

Received by the editors February 11, 2002.

2000 Mathematics Subject Classification. Primary 30C80.

Key words and phrases. Spherical chordal metric, distortion, quasiconformal mappings.

Supported in part by The Academy of Finland, Research Contract 12132. I would also like to thank Matti Vuorinen for pointing out this problem to me as well as for advice and suggestions during the process of writing this paper. 
indicated:

$$
\begin{aligned}
& |f(x)-f(y)| \leq M_{1}(K, n, R)|x-y|^{\alpha}, f\left(B^{n}\right) \subset B^{n}, \\
& |f(x)-f(y)| \leq M_{2}(K, n, R)|x-y|^{\alpha}, f(1)=1, \\
& q(f(x), f(y)) \leq M_{3}(K, n, R) q(x, y)^{\alpha}, f\left(B^{n}\right)=B^{n}, \\
& q(f(x), f(y)) \leq M_{4}(K, n, R) q(x, y)^{\alpha}, f(1)=1,
\end{aligned}
$$

where $\alpha:=K^{1 /(1-n)}$ and $q$ is the spherical metric (defined in (3.1)).

Remark 2.2. For brevity, we will write $M_{1}(K, n):=M_{1}(K, n, 1), M_{2}(K, n):=$ $M_{2}(K, n, 1), M_{3}(K, n):=M_{3}(K, n, \infty)$ and $M_{4}(K, n):=M_{4}(K, n, \infty)$. The two last definitions are to be understood as replacing the condition " $x, y \in B^{n}(R)$ " by the condition " $x, y \in \overline{\mathbb{R}^{n}}$ ".

Remark 2.3. We can also define analogous constants for $n=1$ (see Section 5).

Remark 2.4. Note that for $M_{3}(K, n)$ we require that $f\left(B^{n}\right)=B^{n}$, whereas for $M_{1}(K, n)$ we only have the normalization $f\left(B^{n}\right) \subset B^{n}$. The reason for this discrepancy will become clear in the proof of Theorem 2.8

Lemma 2.5 ([3. pp. 115-117]). The constant $M_{1}(K, n)$ has the following properties:

(1) $M_{1}(K, n) \rightarrow 1$ as $K \rightarrow 1$;

(2) $M_{1}(K, n)$ is bounded for fixed $n$;

(3) $M_{1}(K, n)$ is bounded for fixed $K$.

Moreover, [3] provides explicit estimates that exhibit the described behavior and shows that $M_{2}(K, n)$ has properties (1) and (3), and provides an explicit estimate. In terms of these constants we may state Bonfert-Taylor's result as:

Lemma 2.6 ([2, Lemma 4.1]). $M_{4}(K, 2) \leq 128 \cdot 2^{(1-K) /(2 K)}$.

This result proves property (2) for $n=2$, but not property (1), which is perhaps the most important. The main results of this paper show that (1), (2) and (3) hold for $M_{3}(K, n)$ and (1) and (2) hold for $M_{4}(K, n)$. 3] also shows that $M_{2}(K, n)$ does not satisfy (3). Unfortunately, we were unable to settle whether $M_{4}(K, n)$ satisfies (2). Our results are stated in the following two theorems:

Theorem 2.7. Let $\lambda_{n}$ denote the Grötzsch constant (see [1], (8.38)); let $\eta_{K, n}$ be as in Lemma 3.1 and $\hat{M}_{2}(K, n, R)$ be as in Lemma 4.4. Then

$$
M_{3}(K, n) \leq M_{4}(K, n) \leq \hat{M}_{2}(K, n, R) m^{2 \alpha} \lambda_{n}^{2(1-\alpha)}(1+0.13(1-\alpha)),
$$

where

$$
R:=\sqrt{\frac{1+32^{1-\beta}}{1-32^{1-\beta}}}>1, \quad \beta:=1 / \alpha:=K^{1 /(n-1)}, \quad m:=\eta_{K, n}(1) .
$$

Moreover, $\hat{M}_{2}(K, n, R)$, and therefore $M_{4}(K, n)$, is asymptotically sharp for $K \rightarrow 1$ with this choice of $R$. In particular, for $K \leq 2, M_{4}(K, n)$ satisfies the quite crude estimate:

$$
M_{4}(K, n) \leq m^{4} \lambda_{n}^{4(\beta-1)} e^{0.73 \sqrt{\beta-1}}(1+0.13(1-\alpha)) \leq \exp \{106 \sqrt{K-1}\} .
$$

This means that

$$
q(f(x), f(y)) \leq \exp \{106 \sqrt{K-1}\} q(x, y)^{\alpha}
$$

for $K \leq 2$. If we assume instead that $K \leq 1.01$, then we have the better estimates

$$
M_{4}(K, n) \leq \exp \{7 \sqrt{K-1}\}, \quad q(f(x), f(y)) \leq \exp \{7 \sqrt{K-1}\} q(x, y)^{\alpha} .
$$


Theorem 2.8. The constant $M_{3}(K, n)$ satisfies (2) in Lemma 2.5 In particular,

$$
M_{3}(K, n) \leq 2^{1-\alpha / 2}\left(M_{1}(K, n)\right)^{1+2 \alpha}(1+0.13(1-\alpha)) .
$$

\section{Definitions AND PRELIMINARY RESUlts}

We denote by $\left\{e_{1}(=1), e_{2}(=i), \ldots, e_{n}\right\}$ the standard basis of $\mathbb{R}^{n}$. The following notation will be used:

$$
\begin{array}{rll}
B^{n}(x, r):=\left\{y \in \mathbb{R}^{n}|| x-y \mid<r\right\}, & S^{n-1}(x, r):=\partial B^{n}(x, r), \\
B^{n}(r):=B^{n}(0, r), & S^{n-1}(r):=S^{n-1}(0, r), & B^{n}:=B^{n}(1), \quad S^{n-1}:=S^{n-1}(1) .
\end{array}
$$

We define the spherical metric $q$ by means of the canonical projection onto the Riemann sphere, hence, for $x, y \in \mathbb{R}^{n}$,

$$
q(x, y):=\frac{|x-y|}{\sqrt{1+|x|^{2}} \sqrt{1+|y|^{2}}}, \quad q(x, \infty):=\frac{1}{\sqrt{1+|x|^{2}}} .
$$

We denote the dimension of the Euclidean space under consideration by $n$. Untill Section 5, it is assumed that $n \geq 2$. The letter $K$ will denote the constant of quasiconformality of the arbitrary quasiconformal mapping $f$ (of $\overline{\mathbb{R}^{n}}$ ). More precisely, this entails the following assumptions: $K \in[1, \infty)$ and $f: \overline{\mathbb{R}^{n}} \rightarrow \overline{\mathbb{R}^{n}}$ is an ACL homeomorphism, satisfying

$$
\sup _{|h|=1}\left\{\left|f^{\prime}(x) h\right|\right\}^{n} / K \leq\left|J_{f}(x)\right| \leq K \inf _{|h|=1}\left\{\left|f^{\prime}(x) h\right|\right\}^{n}
$$

a.e. in $\overline{\mathbb{R}^{n}}$, where $J_{f}$ stands for the Jacobian (cf. [1], Theorem 9.9). For ease of reference we define the classes

$$
Q C_{K}:=\left\{f: \overline{\mathbb{R}^{n}} \rightarrow \overline{\mathbb{R}^{n}} \mid f \text { is } K \text {-qc, } f(0)=0, f(1)=1, f(\infty)=\infty\right\}
$$

and refer to mappings of this class as normalized $K$-qc mappings. Note that for $n \geq 3, Q C_{1}$ consists of Euclidean isometries only; that is, reflections in planes containing the $e_{1}$-axis and rotations about the same, by the constancy of the cross ratio under Möbius mappings. From the definition it also follows that if $f \in Q C_{K}$, then $f^{-1} \in Q C_{K}$.

Let $\eta:[0, \infty) \rightarrow[0, \infty)$ be a homeomorphism. Let $D$ and $D^{\prime}$ be domains in $\mathbb{R}^{n}$. We say that a mapping $g: D \rightarrow D^{\prime}$ is $\eta$-quasisymmetric if

$$
\frac{|g(a)-g(c)|}{|g(a)-g(b)|} \leq \eta\left(\frac{|a-c|}{|a-b|}\right)
$$

for all $a, b, c \in D$ with $a \neq b$.

Lemma 3.1 ([1, Theorems 14.6 and 14.8], 7, Theorem 7.47]). Let $\lambda_{n}$ be as in Theorem 2.7. For $n \geq 2$ and $K \geq 1$ there exists a bijection $\eta_{K, n}:[0, \infty) \rightarrow[0, \infty)$ such that every $K$-qc mapping of $\mathbb{R}^{n}$ is $\eta_{K, n}-q s$, and $\eta_{K, n}$ satisfies the following bounds:

(1) $m=\eta_{K, n}(1) \leq \exp \{4 K(K+1) \sqrt{K-1}\}$

(2) $\eta_{K, n}(t) \leq m \lambda_{n}{ }^{1-\alpha} t^{\alpha}$, for $0 \leq t \leq 1$,

(3) $\eta_{K, n}(t) \leq m \lambda_{n}{ }^{\beta-1} t^{\beta}$, for $t \geq 1$,

(4) $\eta_{K, n}{ }^{-1}(t) \geq \lambda_{n}{ }^{1-\beta}(t / m)^{\beta}$, for $0 \leq t \leq m$,

(5) $\eta_{K, n}{ }^{-1}(t) \geq \lambda_{n}{ }^{\alpha-1}(t / m)^{\alpha}$, for $t \geq m$. 
For the remainder of this paper let us write $\alpha:=K^{1 /(1-n)}=: 1 / \beta$ and $m:=$ $\eta_{K, n}(1)$. It is well-known that $\lambda_{n}^{1-\alpha} \leq 2^{1-1 / K} K$ and that $\lambda_{n} \in\left[4,2 e^{n-1}\right]$ (see [1], p. 169).

Lemma 3.2 ([1, 14.36(4)]). For $K \leq 1.01$, we have the better estimate

$$
m=\eta_{K, n}(1) \leq(K-1)^{1-K} e^{9(K-1)} .
$$

\section{Hölder CONTINUity IN THE SPHERICAL METRIC}

The main results, Theorems 2.7 and 2.8, will be proved in this section. To accomplish this, we first temporarily turn to a more general setting. Suppose, therefore, that $g$ is an arbitrary $\gamma$-biHölder continuous mapping with constant $M$ (with respect to the Euclidean metric). What can we say about the Hölder continuity of $g$ with respect to the spherical metric? In this general setting we may state the following lemma, which turns out to be asymptotically sharp for qc-mappings:

Lemma 4.1. For $0<\gamma \leq 1$ consider an arbitrary $\gamma$-biHölder continuous mapping, $g: D \rightarrow D^{\prime}$, fixing the origin (which entails that $D$ and $D^{\prime}$ contain it). That is to say, there exists a constant $M \geq 1$ such that

$$
\left(\frac{|x-y|}{M}\right)^{1 / \gamma} \leq|g(x)-g(y)| \leq M|x-y|^{\gamma},
$$

for all $x, y \in D$. Then $g$ is Hölder continuous with respect to the spherical metric as well, in particular:

$$
q(g(x), g(y)) \leq M^{\prime} q(x, y)^{\gamma}
$$

where $M^{\prime} \leq M^{1+2 \gamma}(1+0.13(1-\gamma))$.

Proof. By 3.1, the second inequality of 4.1 is equivalent to

$$
q(g(x), g(y)) \leq M \sqrt{\frac{\left(1+|x|^{2}\right)^{\gamma}}{1+|g(x)|^{2}}} \sqrt{\frac{\left(1+|y|^{2}\right)^{\gamma}}{1+|g(y)|^{2}}} q(x, y)^{\gamma}
$$

and hence $q(g(x), g(y)) \leq M c q(x, y)^{\gamma}$, where

$$
c:=\sup _{x, g} \frac{\left(1+|x|^{2}\right)^{\gamma}}{1+|g(x)|^{2}}
$$

and the supremum is taken over $x \in D$ and over all mappings $g$ that satisfy the assumptions. Using the first inequality of (4.1) we conclude, by setting $y=0$, that $|g(x)|^{\gamma} M \geq|x|$. Then, since $M \geq 1$ and $0<\gamma \leq 1$, we have

$$
c \leq M^{2 \gamma} \sup _{t \geq 0} \frac{\left(1+t^{\gamma}\right)^{\gamma}}{1+t} \text {. }
$$

To prove that

$$
\frac{\left(1+t^{\gamma}\right)^{\gamma}}{1+t} \leq 1+0.13(1-\gamma)
$$

we take the logarithmic partial derivative, with respect to $\gamma$, of both sides. Since the left and right hand side of (4.2) are equal when $\gamma=1$, we only need to prove that the derivative of the left hand side is greater than that of the right hand side for $0<\gamma<1$. That is, we need to show that

$$
\log (1+s)+\frac{s \log s}{1+s} \geq-\frac{0.13}{1+0.13(1-\gamma)}
$$


where $s:=t^{\gamma}$. It is clear that the right hand side is less than $-0.13 / 1.13<-0.115$.

We conclude the proof by showing that $\log (1+s)+\frac{s \log s}{1+s} \geq-0.1144$. To do this, multiply both sides by $1+s$. This gives the inequality

$$
(1+s) \log (1+s)+s \log s+0.1144(1+s) \geq 0 .
$$

By differentiating it is easy to see that the left hand side has only one minimum which is at $s_{0}=\sqrt{0.25+\exp (-2.1144)}-0.5$. Using a calculator and $s=s_{0}$ we see that this inequality indeed holds.

Remark 4.2. Note that a mapping can be $\gamma$-biHölder continuous in the sense of (4.1) for $\gamma<1$ only if $D$ and $D^{\prime}$ are bounded.

Lemma 4.3. $M_{3}(K, n, 1) \leq M_{1}(K, n)^{1+2 \alpha}(1.13-0.13 \alpha)$.

Proof. The proof follows directly from Lemma 4.1 as $f$ is $\alpha$-Hölder continuous in $B^{n}$ with constant $M_{1}(K, n)$ by definition.

From the Lemmas 2.5 and 4.1 we derive the properties (1)-(3) for $M_{3}(K, n, 1)$. This does not, however, provide the Hölder continuity throughout space. It is possible to remedy this shortcoming as will be shown, but in doing so, we will lose property (2). Therefore we first give an independent proof of property (2).

Proof of Theorem [2.8. By Lemma 4.1 this is clear if $x, y \in \overline{B^{n}}$ or $x, y \in\left\{B^{n}\right\}^{c}$. Let $x \in B^{n}$ and $y \in{\overline{B^{n}}}^{c}$ and $f \in Q C_{K}$ be given. We will prove that there exists a point $w$ in the intersection of the line through $x$ and $y$ with $S^{n-1}$ such that $q(x, w)+q(w, y) \leq \sqrt{2} q(x, y)$. Let $x^{\prime}$ be the image of $x$ in the canonical projection of $\overline{\mathbb{R}^{n}}$ onto $S^{n}$ etc. Then, by definition, $\left|x^{\prime}-w^{\prime}\right|=q(x, w)$ and so on. Let $r$ be the radius of the image of the line containing $x$ and $y$ under the projection. Of the two possible $w^{\prime}$ 's, we chose the one on the shorter arc joining $x^{\prime}$ and $y^{\prime}$. Then we need to show that

$$
r \sqrt{2-2 \cos \alpha}+r \sqrt{2-2 \cos (\beta-\alpha)} \leq 2 r \sqrt{1-\cos \beta}
$$

Let us differentiate the left-hand-side with respect to $\alpha$. We find that the left-handside has a maximum at $\alpha=\beta / 2$. We then have to show that $\sqrt{2-2 \cos \beta / 2} \leq$ $\sqrt{1-\cos \beta}$. However, this follows directly from the equation $\cos \beta=2 \cos ^{2} \beta / 2-1$.

From Lemma 4.1 it follows that

$$
q(f(x), f(w)) \leq M_{3}(K, n, 1) q(x, w)^{\alpha}, \quad q(f(w), f(y)) \leq M_{3}(K, n, 1) q(w, y)^{\alpha} .
$$

Here we have used the fact that a continuous mapping has the same Hölder constant for $B^{n}$ and $\overline{B^{n}}$.

We combine the previous estimates and conclude that

$$
\begin{aligned}
q(f(x), f(y)) & \leq M_{3}(K, n, 1)\left(q(x, w)^{\alpha}+q(w, y)^{\alpha}\right) \\
& \leq M_{3}(K, n, 1) 2^{1-\alpha}(q(x, w)+q(w, y))^{\alpha} \\
& \leq 2^{1-\alpha} M_{3}(K, n, 1)(\sqrt{2} q(x, y))^{\alpha} \\
& =2^{1-\alpha / 2} M_{3}(K, n, 1) q(x, y)^{\alpha} .
\end{aligned}
$$

Thus $M_{3}(K, n) \leq 2^{1-\alpha / 2} M_{3}(K, n, 1)$. Note that this estimate is not asymptotically sharp. 
We now return to deriving an asymptotically sharp estimate and start by presenting an auxiliary lemma stating an explicit relationship between the constant and the domain of definition of the mapping:

Lemma 4.4. For $R \geq 1$ we have

$$
M_{2}(K, n, R) \leq \hat{M}_{2}(K, n, R):=M_{1}(K, n) m \lambda_{n}{ }^{\beta-1} R^{\beta-\alpha} .
$$

Proof. Let $f \in Q C_{K}$ and $x, y \in B^{n}(R)$. Define an auxiliary mapping $g$ by:

$$
g(x):=\eta_{K, n}(R)^{-1} f(R x) .
$$

Since $g\left(B^{n}\right) \subset B^{n}$ it follows from the definition of $M_{1}(K, n)$ that

$$
M_{1}(K, n)|x / R-y / R|^{\alpha} \geq|g(x / R)-g(y / R)|=\eta_{K, n}(R)^{-1}|f(x)-f(y)|,
$$

which is equivalent to $|f(x)-f(y)| \leq M_{1}(K, n) \eta_{K, n}(R) R^{-\alpha}|x-y|^{\alpha}$ and thus, by Lemma 3.1. (4.3) holds with the constant $M_{2}(K, n, R)$ indicated.

Now we will derive a new version of Lemma 4.1, which incorporates the variability of $M_{2}(K, n, R)$ in the proof and is thus specialized to quasiconformal mappings.

Lemma 4.5. Let $f \in Q C_{K}$. Then $\left.f\right|_{B^{n}(R)}$ is Hölder continuous with respect to the spherical metric with constant $M_{4}(K, n, R)$ satisfying

$$
M_{4}(K, n, R) \leq M_{2}(K, n, R) m^{2 \alpha} \lambda_{n}^{2(1-\alpha)}(1+0.13(1-\alpha)) .
$$

Proof. This is proved as Lemma 4.1 except that we will use different methods of estimating the upper bound $c$ of $\left(1+|x|^{2}\right)^{\alpha} /\left(1+|f(x)|^{2}\right)$. If $|f(x)| \geq 1$, we use the estimate

$$
|x| \leq \eta_{K, n}(|f(x)|) \leq m \lambda_{n}{ }^{\beta-1}|f(x)|^{\beta} .
$$

In this case we easily see that $c \leq\left(m \lambda_{n}{ }^{\beta-1}\right)^{2 \alpha}$. For $x \in f^{-1}\left(B^{n}\right)$ we have, by the Hölder continuity of $f^{-1},|x| \leq m \lambda_{n}^{1-\alpha}|f(x)|^{\alpha}$. Then we proceed exactly as in Lemma 4.1 to get $c \leq\left(m \lambda_{n}^{1-\alpha}\right)^{2 \alpha}(1+0.13(1-\alpha))$. It follows that

$c \leq \max \left\{\left(m \lambda_{n}{ }^{\beta-1}\right)^{2 \alpha},\left(m \lambda_{n}^{1-\alpha}\right)^{2 \alpha}(1+0.13(1-\alpha))\right\}=m^{2 \alpha} \lambda_{n}^{2(1-\alpha)}(1+0.13(1-\alpha))$.

Recall that in Lemma 4.1 the Hölder constant in the spherical metric was shown to be less than $M c$ so we are done.

We are now in a position to derive easily the main result.

Proof of Theorem 2.7. By Lemmas 4.4 and 4.5 the estimate holds for $x, y \in B^{n}(R)$. Since the inversion $x \mapsto x|x|^{-2}$ is a $q$-isometry, we conclude that the estimate holds also for $x, y \in B^{n}(1 / R)^{c}$. We now deal with the remaining case: $x \in B^{n}(1 / R)$, $y \in B^{n}(R)^{c}$. Then

$$
q(x, y) \geq \frac{R-1 / R}{\sqrt{1+R^{2}} \sqrt{1+1 / R^{2}}}=\frac{R^{2}-1}{R^{2}+1}=32^{1-\beta} \geq 1 / M_{4}(K, n)^{\beta} .
$$

The last inequality holds, since we may assume (otherwise inequality (2.1) is trivial) $M_{4}(K, n)^{\beta} \geq \lambda_{n}^{3(\beta-1)} \geq 32^{\beta-1}$ since $\lambda_{n} \geq 4$. From this it follows immediately that

$$
M_{4}(K, n) q(x, y)^{\alpha} \geq 1 \geq q(f(x), f(y))
$$

which concludes the proof of (2.1). 
For asymptotical sharpness we still need to prove that $M_{2}(K, n, R) \rightarrow 1$ when $K \rightarrow 1$ for $R$ as given in (2.2), i.e. that $R^{\beta-\alpha} \rightarrow 1$ at the limit. This follows from the following chain of inequalities:

$$
R^{\beta-\alpha} \leq\left(\frac{1+32^{1-\beta}}{1-32^{1-\beta}}\right)^{\beta-1} \leq\left(\frac{2}{1-32^{1-\beta}}\right)^{\beta-1} \leq \exp (0.73 \sqrt{\beta-1}) .
$$

Thus we see that the bound of $M_{4}(K, n)$ is asymptotically sharp.

Remark 4.6. Note how using this latter method does not give us a better explicit bound for $M_{3}(K, n)$ than for $M_{4}(K, n)$. This is a consequence of our operating in Euclidean space, where the sharper restriction of the mappings on the unit circle is not much different from only fixing unity as "viewed" from near infinity. Also note that already for quite small values of $K$, the estimate in Theorem 2.7 is worse than that in the proof of Theorem 2.8 .

Remark 4.7. If $K$ is close to one, we may derive the following bound for $M_{1}(K, n, R)$ in terms of our new constant $M_{3}(K, n, R)$ :

$$
M_{1}(K, n, R) \leq \frac{M_{3}(K, n, R)^{1+2 \alpha}}{(1+R)^{\alpha}-M_{3}(K, n, R)^{2} R^{\alpha}}(1.13-0.13 \alpha) .
$$

It is valid if $(1 / R+1)^{\alpha}>M_{3}(K, n, R)^{2}$. In particular,

$$
M_{1}(K, n) \leq \frac{M_{3}(K, n, 1)}{2^{\alpha}-M_{3}(K, n, 1)^{2}}(1.13-0.13 \alpha) \leq \frac{M_{3}(K, n)}{2^{\alpha}-M_{3}(K, n)^{2}}(1.13-0.13 \alpha)
$$

if $M_{3}(K, n, 1)<2^{\alpha / 2}$. These claims are proved exactly as in Lemma 4.1 so the proofs are omitted here. We see that from bounds on the Hölder constants in the spherical metric we can derive bounds for the constants in the Euclidean metric, as well as the other way around.

\section{An AdDitional Result}

In [5], a one parameter family of $K$-quasisymmetric (qs) functions is defined as those functions $g: \mathbb{R} \rightarrow \mathbb{R}$ that satisfy

$$
\frac{1}{K} \leq \frac{g(x+t)-g(x)}{g(x)-g(x-t)} \leq K
$$

for all $x$ and $t>0$. We will now derive an explicit bound for the Hölder constant in the spherical metric of this class of functions. The proof of this is similar to the existence proof given by Lehto and Virtanen ([5], p. 57), except that the following lemma will be used:

Lemma 5.1 ([4]). Let $f$ be $K$-qs in the sense of [5] with constant $K$. Then $f$ can be extended to an $L(K)$-qc mapping $f: H \rightarrow H$ where

$$
L(K) \leq \min \left\{K^{3 / 2}, 2 K-1\right\}
$$

and $H$ denotes the upper half-plane.

Remark 5.2. We can further extend the mapping of the upper half-plane to a mapping of $\mathbb{R}^{2}$ by reflection.

We will also need the following geometrical lemma, which states quite simply that a point far away from the real axis and from infinity is not near the unit circle in the spherical metric. 


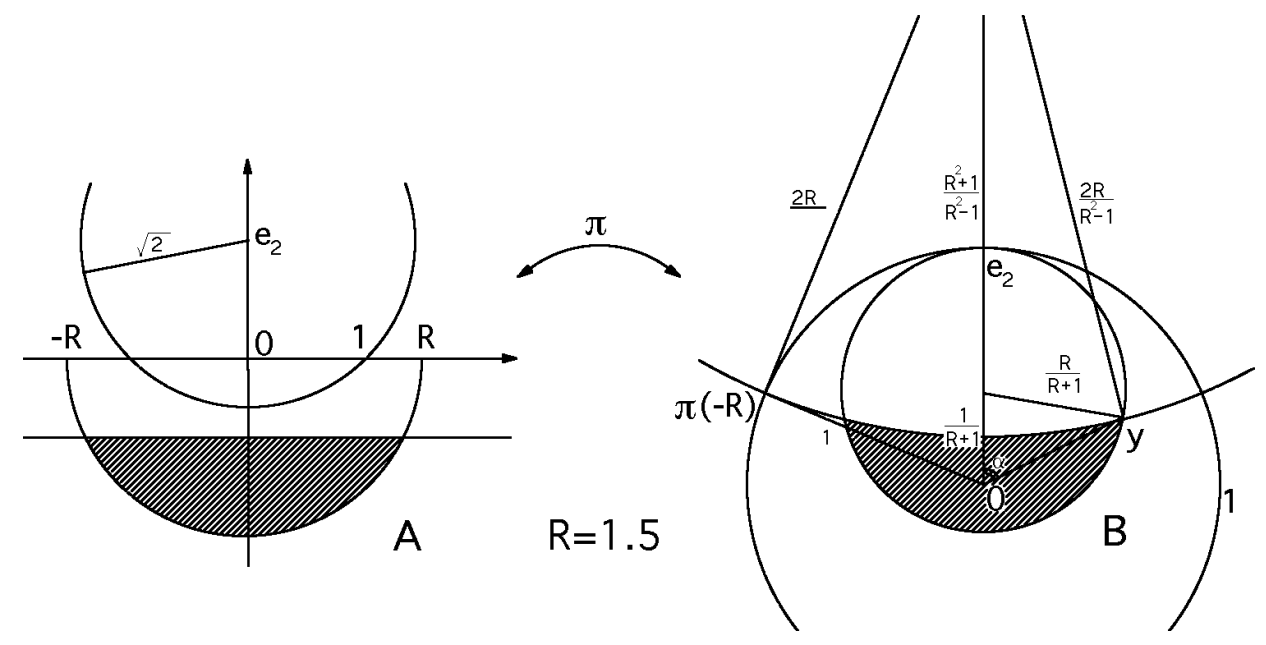

FiguRe 1.

Lemma 5.3. Let $\left(x_{1}, x_{2}\right) \in \mathbb{R}^{2}$ be a point such that $x_{2}<-1 / R$ and $|x| \leq R$ for $R>1$ ( $x$ is in the shaded area in Figure 1A). Let $\pi$ denote the inversion in $S^{1}\left(e_{2}, \sqrt{2}\right)$. Then

$$
|\pi(x)| \leq \sqrt{\frac{R^{3}+R-2}{R^{3}+R+2}} .
$$

Proof. The point $x$ lies in a region bounded by a line and a circle, as shown in Figure 1A. This means that $\pi(x)$ lies in a region bounded by two spheres, as shown in Figure 1B. It is immediately clear that $y$ is as far from the origin as any other point in the region, so that $|\pi(x)| \leq|y|$. We calculate $|y|$ from

$$
\frac{R^{2}}{(R+1)^{2}}=\frac{1}{(R+1)^{2}}+|y|^{2}-2 \frac{|y|}{R+1} \cos \alpha
$$

and

$$
\frac{2 R^{2}}{\left(R^{2}-1\right)^{2}}=\frac{\left(R^{2}+1\right)^{2}}{\left(R^{2}-1\right)^{2}}+|y|^{2}-2|y| \frac{\left(R^{2}+1\right)}{\left(R^{2}-1\right)} \cos \alpha .
$$

(Note that here $\alpha$ refers to an angle as indicated in the picture, not to $K^{1 /(1-n)}$.) We subtract the second equation from the first to get

$$
2|y| \cos \alpha=2 R \frac{R-1}{R^{2}-R+2} .
$$

Now using this in the first equation gives us the desired formula

$$
|y|^{2}=\left(R^{3}+R-2\right) /\left(R^{3}+R+2\right) \text {. }
$$

Theorem 5.4. Let $f: \mathbb{R} \rightarrow \mathbb{R}$ be K-qs. Then

$$
q(f(x), f(y)) \leq(1 / 2) 16^{1-1 / L}\left(R^{3}+R+\sqrt{\left(R^{3}+R\right)^{2}-4}\right) q(x, y)^{1 / L}
$$

for all $x, y \in \mathbb{R}$, where $R:=\eta_{L, 2}(1)$ and $L \leq \min \left\{K^{3 / 2}, 2 K-1\right\}$. 
Proof. Denote the symmetric $L$-qc extension of $f$ to a mapping from $\mathbb{R}^{2}$ to $\mathbb{R}^{2}$ by $f$, again (here we use Lemma 5.1). We assume without loss of generality that $f(0)=0$ and $f(1)=1$. Since $f$ is $L$-qc, $\left|f\left(-e_{2}\right)-f(0)\right| \leq \eta_{L, 2}(1)$. We may assume without loss of generality that $f\left(-e_{2}\right)_{2}<0$. Let $x_{0}$ be a point, such that the projection of $f\left(-e_{2}\right)$ in the real axis equals $f\left(x_{0}\right)$. Then

$$
\left|f\left(-e_{2}\right)-f\left(x_{0}\right)\right| \geq \eta_{L, 2}^{-1}\left(\left|e_{2}+x_{0}\right|\right) \geq \eta_{L, 2}^{-1}(1)=1 / \eta_{L, 2}(1),
$$

where the last inequality holds, since $\eta_{L, 2}^{-1}$ is increasing and $\left|e_{2}+x_{0}\right| \geq 1$, since $x_{0} \in \mathbb{R}$ and the equality holds by [1], Remark 9.41 (1). Therefore we may take $R=\eta_{L, 2}(1)$ in Lemma 5.3 to conclude that

$$
\left|\pi\left(f\left(-e_{2}\right)\right)\right| \leq \sqrt{\frac{R^{3}+R-2}{R^{3}+R+2}}
$$

with $\pi$ the inversion in $S^{1}\left(e_{2}, \sqrt{2}\right)$. Let $g:=\pi \circ f \circ \pi$. Since $\pi$ is a 1 -qc, $g$ is $L$-qc. Since $f$ fixes $\mathbb{R}, g$ fixes $S^{1}$. However, (5.1) means that $|g(0)| \leq$ $\sqrt{R^{3}+R-2} / \sqrt{R^{3}+R+2}$, since $\pi\left(-e_{2}\right)=0$.

Let $\tau$ be a Möbius transformation with $\tau\left(S^{1}\right)=S^{1}$ and $\tau(g(0))=0$. Then, since $g$ is symmetric in $S^{1}, \tau(g(\infty))=\infty$. Now $\tau$ is biLipschitz with constant

$$
\frac{1+|g(0)|}{1-|g(0)|} \leq \frac{\sqrt{R^{3}+R-2}+\sqrt{R^{3}+R+2}}{\sqrt{R^{3}+R+2}-\sqrt{R^{3}+R-2}}=\frac{1}{2}\left(R^{3}+R+\sqrt{\left(R^{3}+R\right)^{2}-4}\right) .
$$

(See e.g. [7], chapter 1, for these elementary Möbius mapping results.) Since $\tau \circ g$ is $L$-qc and fixes $S^{1}, 0$ and $\infty$, we have $|\tau(g(x))-\tau(g(y))| \leq 16^{1-1 / L}|x-y|^{1 / L}$, for $x, y \in S^{1}$ by Theorem 15.5 of [1]. It follows from the biLipschitz property of $\tau$ that

$$
|g(x)-g(y)| \leq(1 / 2) 16^{1-1 / L}\left(R^{3}+R+\sqrt{\left(R^{3}+R\right)^{2}-4}\right)|x-y|^{1 / L} .
$$

When we set $x=\pi\left(x^{\prime}\right)$ and $y=\pi\left(y^{\prime}\right)$, we get

$$
q\left(f\left(x^{\prime}\right), f\left(y^{\prime}\right)\right) \leq(1 / 2) 16^{1-1 / L}\left(R^{3}+R+\sqrt{\left(R^{3}+R\right)^{2}-4}\right) q\left(x^{\prime}, y^{\prime}\right)^{1 / L},
$$

for $x^{\prime}, y^{\prime} \in \mathbb{R}$, which is what we wanted to show.

Remark 5.5. Since $R \leq e^{\pi(L-1 / L)}$ by Theorem 10.35 of [1], we readily get explicit estimates from the above formula. Note that

$$
(1 / 2) 16^{1-1 / L}\left(R^{3}+R+\sqrt{\left(R^{3}+R\right)^{2}-4}\right) \rightarrow 1
$$

as $K \rightarrow 1$.

Remark 5.6. The previous theorem could be extended to higher dimensions by using the extension result of [6] instead of that of [4], which would allow us to derive estimates similar to those in the beginning of this paper. This does not, however, give the explicit results that we have strived for.

\section{REFERENCES}

1. G. D. Anderson, M. K. Vamanamurthy and M. Vuorinen: Conformal Invariants, Inequalities, and Quasiconformal Maps, J. Wiley, New York, 1997. MR 98h:30033

2. P. Bonfert-Taylor: Jørgensen inequality for discrete convergence groups, Ann. Acad. Sci. Fenn. Math. 25 (2000), no. 1, 131-150. MR 2001a:30056

3. R. Fehlmann and M. Vuorinen: Mori's Theorem for n-Dimensional Quasiconformal Mappings, Ann. Acad. Sci. Fenn. Ser. A I Math. 13 (1988), no. 1, 111-124. MR 90a:30060

4. M. Lehtinen: Remarks on the maximal dilatation of the Beurling-Ahlfors extension, Ann. Acad. Sci. Fenn. Ser. A I Math. 9 (1984), 133-139. MR 85j:30039 
5. O. Lehto and K. I. Virtanen: Quasiconformal Mappings of the Plane, $2^{\text {nd }}$ ed., Grundlehren der Mathematischen Wissenschaften, Band 126, Springer Verlag, Berlin-Heidelberg-New York, 1973. MR 49:9202

6. P. Tukia And J. VÄisäLÄ: Quasiconformal extensions from dimension $n$ to $n+1$, Ann. of Math. 115 (1982), 331-342. MR 84i:30030

7. M. Vuorinen: Conformal Geometry and Quasiregular Mappings, Lecture Notes in Mathematics 1319, Springer-Verlag, Berlin-Heidelberg-New York, 1988. MR 89k:30021

Department of Mathematics, P.O. Box 4, 00014 University of Helsinki, Finland

E-mail address: peter.hasto@helsinki.fi 